\title{
UNSUPERVISED STATISTICAL SKETCHING FOR NON-PHOTOREALISTIC RENDERING MODELS
}

\author{
Max Mignotte \\ DIRO, Département d'Informatique et de Recherche Opérationnelle, CP 6128, \\ Succ. Centre-Ville, P.O. 6128, Montréal (Québec), H3C 3J7. \\ HTTP : WWW.IRO.UMONTREAL.CA/ MIGNOTTE/ \\ E-MAIL : MIGNOTTE@IRO.UMONTREAL.CA
}

\begin{abstract}
This paper investigates the use of the Bayesian inference for devising an unsupervised sketch rendering procedure. As likelihood model of this inference, we exploit the recent statistical model of the gradient vector field distribution proposed by Destrempes et al. for contour detection. A global prior deformation model for each pencil stroke is also considered. In this Bayesian framework, the placement of each stroke is viewed as the search of the Maximum A Posteriori estimation of the posterior distribution of its deformations. We use a stochastic optimization algorithm in order to find these optimal deformations. This yields an unsupervised method to create realistic hand-sketched pencil drawings. Combined with an example-based local rendering model, used to transfer the textural tone value of a given depiction style, the proposed scheme allows to simulate automatic synthesis of various artistic illustration styles ${ }^{\dagger}$.
\end{abstract}

\section{INTRODUCTION}

In the past few years, there has been a great deal of research works in creating artistic styles by computer. This field of research is known as Non-Photorealistic Rendering (NPR). These methods refer to any image processing techniques which can transform an image into a specific artistic styles (or more precisely that suggest an artistic style) such as ink painting, charcoal drawing, engraving, etc., or, more generally, in a style other than realism (see [1] for a excellent review of existing NPR techniques).

Among them, freehand sketch style remains especially appealing as artistic depiction style. It can be used for illustrative purposes or artistic effects, but this representation style is also interesting due to its appealing ability to abstract away detail, to clarify or simplify shapes and to focus the viewer's attention on relevant features. In addition, this depiction style allows to efficiently convey a great deal of information with very few pencil strokes.

There are many commercial softwares [2,3] or previous works $[4,5,6]$ which propose (widely) supervised systems in which the user has to place each individual stroke manually and in which the system control over the tone, the smudging and amount of details or systems that use a 3D polygonal mesh information [7]. None considered an unsupervised system for pencil stroke placement allowing to create automatic realistic hand-sketched pencil drawing directly from a 2D grey-scale real image. Let us add that the methods proposed by the computer graphics community are generally

\footnotetext{
${ }^{1} \dagger$ cf. http : www.iro.umontreal.ca/ mignotte/ICIP03/ for additional and color Non-Photorealistic Rendering results.
}

specifically tailored to a particular rendering style and/or are purely deterministic algorithmic techniques (excepted $[8,9]$ ) and not statistical.

The main contribution of this paper is to provide a Bayesian formulation for the sketch rendering problem. This model exploits a recent likelihood model based on the statistical distribution of the gradient vector field introduced in [10] and a global prior deformation model for each pencil stroke. This global Bayesian sketch rendering model can also be efficiently combined with the nonparametric (and example-based) Bayesian local rendering model proposed in [11]. This example-based rendering model can also be used to transfer the tonal adjustment or the texture of a given artistic (rendering) depiction style. Combination of these two techniques allows to create various artistic illustration styles.

This paper is organized as follows. In Sections 2 and 3 we present the prior and likelihood models of our Bayesian sketch rendering method. Sections 4 and 5 describe the localization and rendering procedures of a pencil stroke. Finally, obtained results of our freehand sketching method are presented and combined with the sample-based rendering model proposed in [11].

\section{PRIOR MODEL \& DISTRIBUTION}

\subsection{Prior Model}

In our model, a pencil stroke is represented by a template, i.e., a set of $\tau$ labeled points $\gamma=\left(x_{1}, y_{1}, \ldots, x_{\tau}, y_{\tau}\right)$ equally sampled, which approximate the outline of a straight line (see Fig. 1). A cubic B-spline shape representation involving these $\tau$ control points is then defined. In order to take into account the variability of the pencil stroke deformations, we introduce on this template, a set of admissible global non-affine and affine deformations.

\section{Non-Affine Deformations}

This deformation model was first proposed by Jain et al. [12] for the localization of shapes. In this approach, the template image is considered to be mapped to the unit square $S=[0,1]^{2}$. The deformation is then represented by a displacement function denoted $\mathcal{D}(x, y)$. The mapping $(x, y) \mapsto(x, y)+\mathcal{D}(x, y)$ is thus a deformation of $S$, a smooth mapping of the unit square onto itself. The space of such displacement functions is spanned by the following orthogonal bases,

$$
\begin{aligned}
& e_{m, n}^{x}(x, y)=(2 \sin (\pi n x) \cos (\pi m y), 0), \\
& e_{m, n}^{y}(x, y)=(0,2 \cos (\pi n x) \sin (\pi m y)),
\end{aligned}
$$


for $m, n=1,2, \ldots$ Low values of $m$ and/or $n$ correspond to lower frequency components of the deformation in the $x$ and $y$ directions, respectively. The displacement function is then chosen as follows,

$$
\mathcal{D}_{\xi}(x, y)=\sum_{m=1}^{M} \sum_{n=1}^{N} \frac{\xi_{m, n}^{x} e_{m, n}^{x}(x, y)+\xi_{m, n}^{y} e_{m, n}^{y}(x, y)}{\lambda_{m, n}},
$$

where $\lambda_{m, n}=\pi^{2}\left(n^{2}+m^{2}\right)$ are the normalizing constants. The parameter vectors $\xi$ are the projections of the displacement function on the orthogonal basis previously defined. In order to allow a sufficiently wide range of possible deformations, while keeping the number of parameters reasonable, we use $M=N=2$. Fig. 1 illustrates the series of deformations of an initial template using higher order terms and different values of $M$ and $N$. We can notice that these deformations can efficiently express the inherent variability of a pencil stroke and that the deformation becomes more complex as higher frequency components are added to the displacement function $\mathcal{D}$.

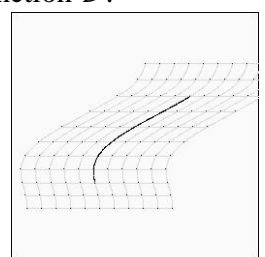

(a)

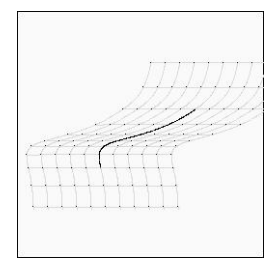

(b)
FIG. 1 - Global non-affine deformations, (a) $\xi_{1,2}^{x}=-4, \xi_{2,2}^{x}=$ $-1, \xi_{1,0}^{y}=4$. (b) by adding $\xi_{2,2}^{y}=-4$.

\section{Affine Deformations}

Finally, we introduce a set of admissible linear and rigid deformations on $\gamma$. These deformations involve translation $\left(\tau_{x}, \tau_{y}\right)$, scaling $s$, and rotation $\psi$ applied point-wise to the template.

Let now $\gamma_{\theta}$ be a deformed version of the original prototype $\gamma_{0}$ according to the aforementioned deformations with parameter vector $\theta$. A global configuration of the deformable template is thus described by four parameters corresponding to affine transformations, and four global non affine deformation parameters (due to the symmetry of the non-deformed original template associated to the pencil stroke), i.e., $\left(\xi_{1,2}^{x}, \xi_{2,2}^{x}, \xi_{1,0}^{y}, \xi_{2,1}^{y}\right)$. The prototype template along with the set of aforementioned deformations $\theta$ constitute our global prior model of a pencil stroke.

\subsection{Prior Distribution}

Let $\Theta$ be the random variable corresponding to the vector of (affine and non-affine) deformations. As proposed by Jain et al. [12], we model the distribution of $\Theta$ by,

$$
P_{\Theta}(\theta) \propto \mathcal{U}\left(\tau_{x}, \tau_{y}, s, \psi\right) \exp (-\rho \underbrace{\sum_{\xi_{i} \in \xi}\left(\left(\xi_{i}^{x}\right)^{2}+\left(\xi_{i}^{y}\right)^{2}\right)}_{U_{p}(\xi)}) .
$$

where $\mathcal{U}$ designates the uniform distribution and where the parameter $\rho$ allows to control the "rigidity" of the pencil stroke. $\xi$, the set of possible deformation parameter, allows to control the complexity of the used admissible deformations.

Let us add that the Bayesian sketch model we propose is also suitable for an example-based deformation model of pencil stroke from a sample of pencil strokes extracted from a real freehand sketch drawing. This can be done in a training step by using a Probabilistic Principal Component Analysis or a mixture of PPCA [13] allowing to learn the principal non-linear deformations (and the prior distribution associated to these deformations) of the stroke shape preferentially used by the artist of a freehand drawing. Nevertheless, reliable detection of individual strokes in a freehand drawing, necessary to constitute the learning set, is not an easy task and can only be done with supervision.

\section{LIKELIHOOD MODEL \& DISTRIBUTION}

We base the likelihood of deformations of a given (candidate) pencil stroke on the statistical distribution of the gradient vector field (norm and angle) of the gray levels. Already applied for the unsupervised detection of contours [10] and the localization of complex shape [14], this model can define, with a true statistical meaning, the likelihood of a deformation $\theta$ (or equivalently a pencil stroke template $\gamma_{\theta}$ with deformation parameters $\theta$ ) by the following Gibbs distribution,

$$
P_{Y / \Theta}(y / \theta)=\frac{1}{Z_{y}} \exp \left(-U_{l}(\theta, y, \gamma)\right)
$$

where $Y$ represents the field of observation, i.e., the set of random variable couples associated to the gradient vector (norm and angle) of each pixel of the input image. $Z_{y}$ is a normalizing constant depending on the observable data $y$ only and $\gamma$ designates the parameter vector of this likelihood distribution, estimated in a Maximum Likelihood (ML) sense in a preliminary training step (see [10] for more details).

\section{STROKE LOCALIZATION}

We deduce from above and from Bayesian inference the posterior distribution of a deformed pencil stroke given the observable data,

$$
\begin{aligned}
P_{\Theta / Y}(\theta / y) & \propto P_{Y / \Theta}(y / \theta) P_{\Theta}(\theta), \\
& =\exp \left(-\left(U_{l}(\theta, y, \gamma)+\rho U_{p}(\xi)\right)\right), \\
& =\exp (-U(\theta, y, \gamma)) .
\end{aligned}
$$

Where $U_{l}$ s the likelihood energy term and $U_{p}$ the prior energy term. We view the localization of a pencil stroke in a sketch drawing as finding its deformation $\theta$ that maximizes the posterior distribution $P_{\Theta / Y}(\theta / y)$ or equivalently as a the search of the Maximum A Posteriori estimation of $\theta, \hat{\theta}_{\mathrm{MAP}} \in \arg \min _{\theta} U(\theta, y, \gamma)$.

The search of $\hat{\theta}_{\text {MAP }}$ is equivalent to the minimization of $U$. Our assumption is that an optimal solution for the energy function $U$ corresponds to a good location, orientation, scale and non-affine deformation of a pencil stroke.

The objective function $U(\theta, y, \gamma)$ to be minimized is complex with several local extrema over the deformation parameter space. In order to solve it efficiently, we resort to the (evolutionary) stochastic optimization algorithm described in [15]. In order to speed up the convergence rate, we proceed in the following manner,

\section{Initialization Step}

First, we use a Canny edge detector [16] to estimate the edge map of the input image. We exploit one edge point of this Canny edge detection out of $x$ ( $x=3$ in our application) edge points as a set of potential location $\left(\tau_{x}, \tau_{y}\right)$ of a pencil stroke. 


\section{Local Optimization}

For each $N_{w} \times N_{w}$ non-overlapping sliding window over the input image, we select one pencil stroke in the following manner,

1. For each potential location $\left(\tau_{x}, \tau_{y}\right)$ within this window and defined in the initialization step, we use the optimization algorithm described in [15] in order to minimize the Gibbs field $U$ on a restricted domain defined by,

$$
\begin{aligned}
\frac{N_{i}}{13} \leq \quad s & \leq \frac{N_{i}}{3} \\
\psi\left(\tau_{x}, \tau_{y}\right)-\frac{\pi}{4} & \leq \quad \psi \quad \leq \psi\left(\tau_{x}, \tau_{y}\right)+\frac{\pi}{4} \\
-5 & \leq \xi_{m, n} \leq 5, \quad \forall m, n
\end{aligned}
$$

where $N_{i} \times N_{i}$ is the size of the input image and $\psi\left(\tau_{x}, \tau_{y}\right)$ designates the gradient orientation at $\left(\tau_{x}, \tau_{y}\right)$.

2. We keep the configuration of the pencil stroke that is the most likely i.e., the deformation of the pencil stroke associated to the lowest Gibbs energy.

\section{PENCIL STROKE \& SKETCH RENDERING}

The first enhancement we propose is to render each pencil stroke with variable width and pressure. In our model, the stroke thickness and pressure first depends on the considered depiction style and is also considered to be linearly inversely proportional to the Gibbs energy (i.e., a less probable stroke is drawn with a low thickness and pressure and inversely).

The second enhancement is to simulate a given texture for the stroke (e.g., charcoal, sanguine, graphite pencil, etc.). In our application, the texture of a stroke is rendered by the non-parametric (and example-based) local rendering model proposed in [11]. This example-based model can also be used to transfer the tonal adjustment (or the texture created by the smudging effect in regions where there is no detected contours and thus no pencil stroke placement) of a given artistic rendering depiction style.

\section{EXPERIMENTAL RESULTS}

The prototype template of a pencil stroke is a B-spline connected 12-points model. The gradient vector field probability map and its parameters are given by [10]. For the Canny edge detector, the lower and upper thresholds are given by the estimation technique proposed in [16]. This estimation relies on a single threshold determining the percentage of edges to be found. This last threshold and the size of the non-overlapping sliding window $N_{w}$ determine the number of strokes of the sketch drawing. The weighting factor $\rho$ penalizing the prior term $U_{p}$ with respect to the likelihood term $U_{l}$ is set to 0.1. The Diameter of the exploration graph of the $E / S$ algorithm are set equal to $D=32$ and the size of the population is $n=20$. These two control parameters are classically used and are independent of the energy function to be minimized [15]. We consider 1000 iterations of the $E / S$ optimization procedure for each local optimization procedure. In total, the procedure takes 20 minutes for a sketch drawing on a $1.2 \mathrm{GHz} \mathrm{PC}$ workstation running Linux.

Several examples of sketch drawing results are shown in Figs. 2. In these examples we use the local rendering model proposed in [11] to simulate a charcoal texture for each pencil stroke. Several examples of sketch rendering results, combined with an example-based local rendering model, used to transfer the textural tone value of a given depiction style, are also shown in Fig. 3 and Figs. 4. The proposed scheme allows to simulate automatic synthesis of various artistic illustration styles such as the charcoal, sanguine and graphite pencil style ${ }^{\dagger}$ see http : www.iro.umontreal.ca $/ \sim$ mignotte/ICIP03/ for additional and color rendering results).

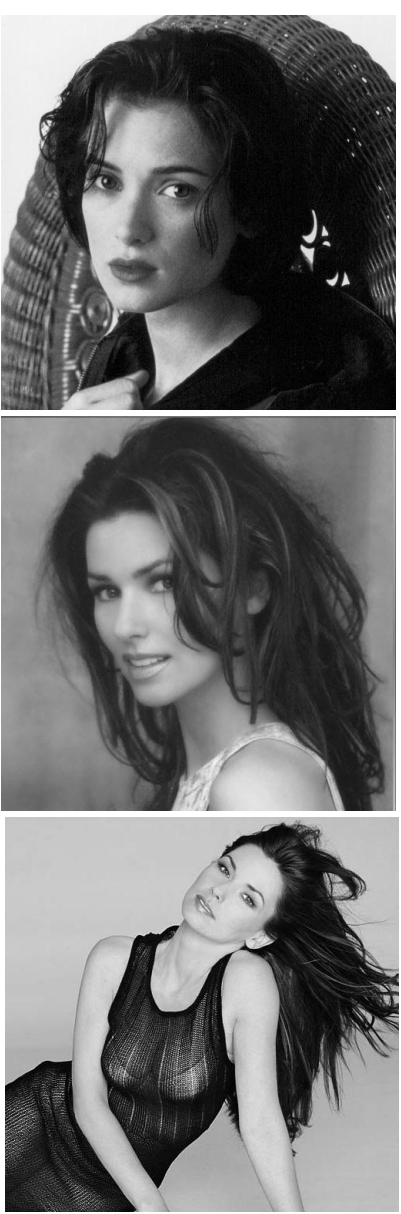

(a)

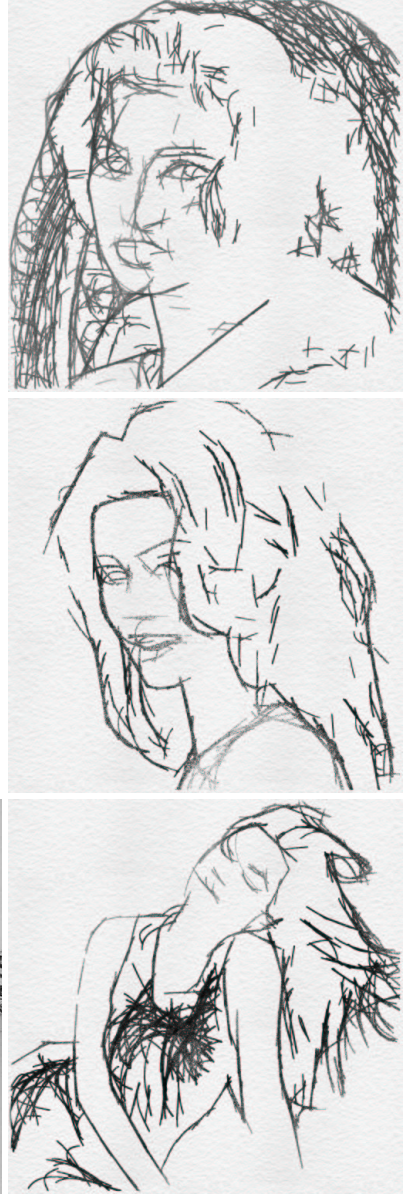

(b)
FIG. 2 - (a) Real picture, (b) Examples of sketch drawing with a pencil stroke simulating the graphite (top) and charcoal style (middle and bottom $)^{\dagger}$.

\section{CONCLUSION}

In this paper, we have presented a statistical method for the free-hand sketch rendering problem. This model is based on a likelihood model of the gradient vector field distribution and a simple parametric prior deformation model for each pencil stroke. The model we propose is also suitable for an example-based deformation model of pencil stroke by a a Probabilistic Principal Component Analysis or a mixture of PPCA [13]. This method would allow to learn the principal non-linear deformations (and the prior distribution associated to these deformations) of the stroke shapes preferentially used by an artist in a given freehand drawing. The global Bayesian parametric sketch rendering model we propose can also be efficiently combined with the local Bayesian non parametric rendering model proposed in [11] in order to enhance the sketch drawing by simulating the textural tone value or the smudging process of a given artistic rendering depiction style. These two complementary schemes allow to simulate automatic synthesis of various artistic illustration styles. 




FIG. 3 - Example of sketch drawing combined with an examplebased local rendering model. From top to bottom, real drawing sample used to train the local rendering model ([11]) with the black ink style, sketch drawing with the textural tone value generated by this local rendering model ${ }^{\dagger}$.

\section{REFERENCES}

[1] C. Reynolds. Stylized depiction in computer graphics, NPR, painterly and toon rendering. http ://www.red3d.com/cwr/npr/.

[2] Fractal design corporation. Fractal design Painter.

[3] Adobe systems. Photoshop 5.0.

[4] F. Durand, V. Ostromoukhov, M. Miller, F. Duranleau, and J. Dorsey. Decoupling strokes and high-level attributes for interactive traditional drawing. In Proceedings of the 12th Eurographics Workshop on Rendering, pages 71-82, August 2001.

[5] M.P. Salisbury, M.T. Wong, J.F. Hughes, and D.H. Salesin. Orientable textures for image-based pen-and-ink illustration. In Proceedings of SIGGRAPH'97, pages 401-406, August 1997.

[6] M.P. Salisbury, S.E. Anderson, R. Barzel, and D.H. Salesin. Interactive penand-ink illustration. In Proceedings of SIGGRAPH'94, pages 101-108, July 1994.

[7] L. Markosian, M. A. Kowalski, S. J. Trychin, L.D. Bourdev, D. Goldstein, and J.F. Hughes. Real-time nonphotorealistic rendering. In Proceedings of SIGGRAPH'97.

[8] T. Szirányi and Z. Tóth. Optimization of paintbrush rendering of images by dynamic mcmc methods. In Lecture Notes on Computer Science, volume 2134, pages 201-215, 2001

[9] T. Szirányi and Z. Tóth. Random paintbrush transformation. In 15th ICPR'00, volume 3, pages $155-158,2000$.

[10] F. Destrempes and M. Mignotte. Unsupervised detection and semi-automatic extraction of contours using a statistical model and dynamic programming. In 4th IASTED International Conference on Signal and Image Processing, ICSIP'02, pages 66-71, August 2002.

[11] M. Mignotte. Bayesian rendering with non-parametric multiscale prior model. In 16th IEEE International Conference on Pattern Recognition, ICPR'02, volume 1, pages 247-250, August 2002.

[12] A.K. Jain, Y. Zhong, and S. Lakshmanan. Object matching using deformable templates. IEEE Trans. on Pattern Analysis and Machine Intelligence, 18(3) :267-278, 1996.

[13] M. E. Tipping and C. M. Bishop. Mixtures of probabilistic principal componen analyzeers. Neural Computation, 11(2) :443-482, 1999.

[14] F. Destrempes and M. Mignotte. Unsupervised localization of shapes using statistical models. In 4th IASTED International Conference on Signal and Image Processing, ICSIP'02, pages 60-65, August 2002.

[15] O. François. An evolutionary strategy for global minimization and its Markov chain analysis. IEEE Trans. on Evolutionary Computation, 2(3) :77-90, 1998.

[16] J. Canny. A computational approach edge detection. IEEE Trans. on Pattern Analysis and Machine Intelligence, 8(6):679-697, 1986.

$1 \uparrow$ cf. http : www.iro.umontreal.ca/ mignotte/ICIP03/ for additional and color Non-Photorealistic Rendering results.
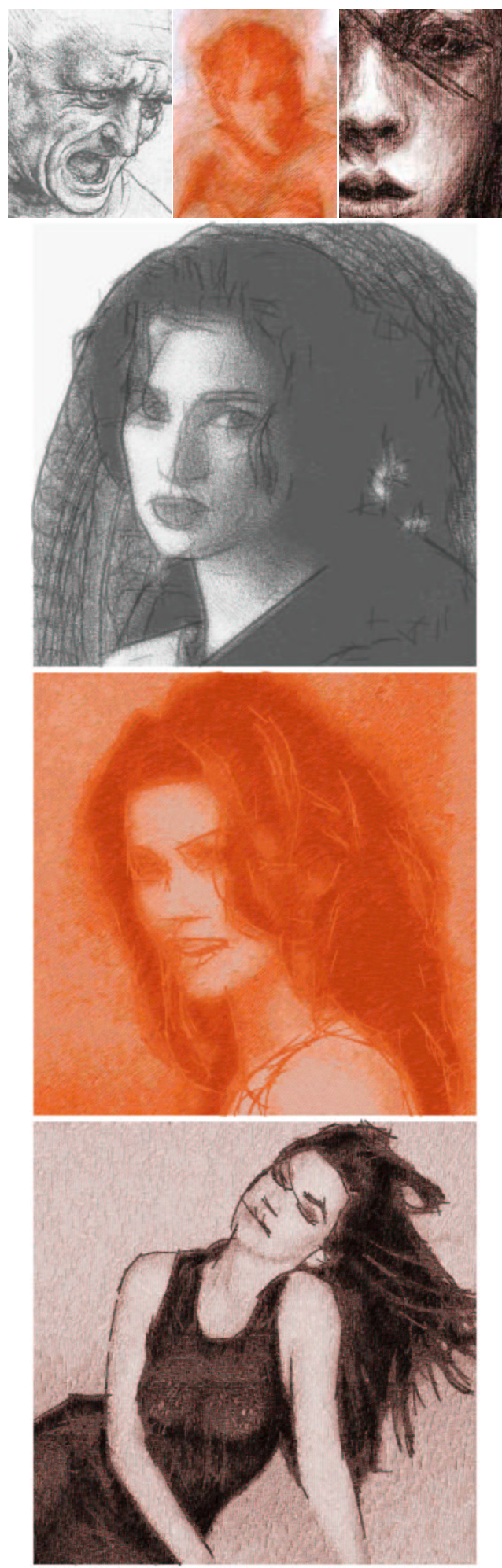

FIG. 4 - Examples of sketch drawing combined with an examplebased local rendering model. From top to bottom and left to right, real drawing sample used to train the local rendering model ([11]) with respectively the graphite pencil, sanguine and charcoal style, sketch drawing with the textural tone value generated by each local rendering model with the above-mentioned style ${ }^{\dagger}$. 